\title{
Compostos poliméricos como aditivos de argilas bentoníticas
}

\section{(Polymeric compounds as additives for bentonite clays)}

\author{
M. I. R. Barbosa ${ }^{\text {, L. V. Amorim }}$, H. C. Ferreira \\ Unidade Acadêmica de Engenharia de Materiais, Laboratório de Referência em Dessalinização - LABDES \\ Universidade Federal de Campina Grande - UFCG \\ Av. Aprígio Veloso 882, Bodocongó, Campina Grande, PB 58109-970 \\ ingridroch@gmail.com
}

\begin{abstract}
Resumo
Este trabalho tem por objetivo avaliar o efeito da utilização de compostos poliméricos na aditivação de bentonitas de Boa Vista, PB, consideradas de qualidade inferior, para utilização em fluidos de perfuração à base de água, visando a melhoria das suas propriedades reológicas e de filtração. Foram estudadas uma amostra de argila bentonítica e três amostras de aditivos poliméricos, sendo uma de poliacrilamida parcialmente hidrolisada e duas de carboximetilcelulose de diferentes graus de viscosidade. O dimensionamento das composições do composto polimérico foi obtido por meio da metodologia de delineamento de misturas, sendo definidas dez composições. O composto polimérico foi misturado à argila seca, em diferentes concentrações e, em seguida, preparados os fluidos de perfuração e determinados as viscosidades aparente e plástica, o limite de escoamento e o volume de filtrado segundo normas da Petrobrás. Os resultados mostram que a aditivação da argila bentonítica com o composto polimérico melhora as suas propriedades, adequando-a às especificações da Petrobrás para utilização em fluidos de perfuração à base de água.

Palavras-chave: bentonitas, compostos poliméricos, fluidos de perfuração, propriedades reológicas.
\end{abstract}

\begin{abstract}
The aim of this work is to evaluate the effect of polymeric compound on the treatment of bentonite clays for using in water and clay based drilling fluids for the improvement of the filtration and the rheological properties. One industrialized sodic bentonite clay sample and three polymeric additive samples, being two samples of carboximethylcellulose and one of partially-hydrolyzed polyacrylamide, were studied. The compositions of the polymeric compound were obtained through the methodology of mixture design, being defined ten different compositions. The polymeric compound with dry clay was mixed in different concentrations and the drilling fluids were prepared according to the Petrobrás standards; the apparent and plastic viscosities, the yield stress and the water loss were determined. The results show that the use of additives in bentonite clays with polymeric compound improves their properties, according to the Petrobrás specifications for their use in water based drilling fluids.
\end{abstract}

Keywords: bentonite clays, polymeric compounds, drilling fluids, rheological properties.

\section{INTRODUÇÃO}

A bentonita é uma argila largamente utilizada em muitos setores da indústria, tais como perfuração de poços de petróleo e de água, operações de sondagem, fundições diversas, pelotização de minério de ferro, indústria química e farmacêutica, entre outros [1]. É caracterizada pela predominância dos argilominerais do grupo da esmectita, ilita e caulinita [1], sendo constituída por duas folhas tetraédrica de silicatos separadas por uma folha octaédrica de alumina, unidas por oxigênios comuns às folhas (Fig. 1) [2]. No espaço entre as camadas encontramse as moléculas de água adsorvidas e os chamados cátions trocáveis, que podem ser $\mathrm{Ca}^{2+}, \mathrm{Mg}^{2+}$ e $\mathrm{Na}^{+}$. Nas argilas

${ }^{1}$ Programa de Pós-Graduação em Ciência e Engenharia de Materiais - Bolsista ANP/PRH-25

${ }^{2}$ Pesquisadora Visitante ANP/PRH-25 bentoníticas policatiônicas, estão presentes os três cátions citados acima [3].

As argilas bentoníticas policatiônicas, quando transformadas em sódicas pelo tratamento com carbonato de sódio $\left(\mathrm{Na}_{2} \mathrm{CO}_{3}\right)$, incham na presença de água, aumentando várias vezes seu volume inicial. Isto é possível porque a presença do $\mathrm{Na}^{+}$como cátion trocável predominante permite a adsorção de várias moléculas de água, aumentando a distância interplanar entre as camadas, separando as partículas de argilas umas das outras. Essa distância interplanar pode atingir valores superiores a 40,0 Å. Nas argilas policatiônicas, há uma limitação na quantidade de água adsorvida, fazendo com que as partículas permaneçam unidas umas às outras por interações elétricas e de massa [2, 3].

Quando transformadas em sódicas, as argilas bentoníticas podem ser utilizadas no preparo de fluidos de perfuração devido as excelentes propriedades coloidais 


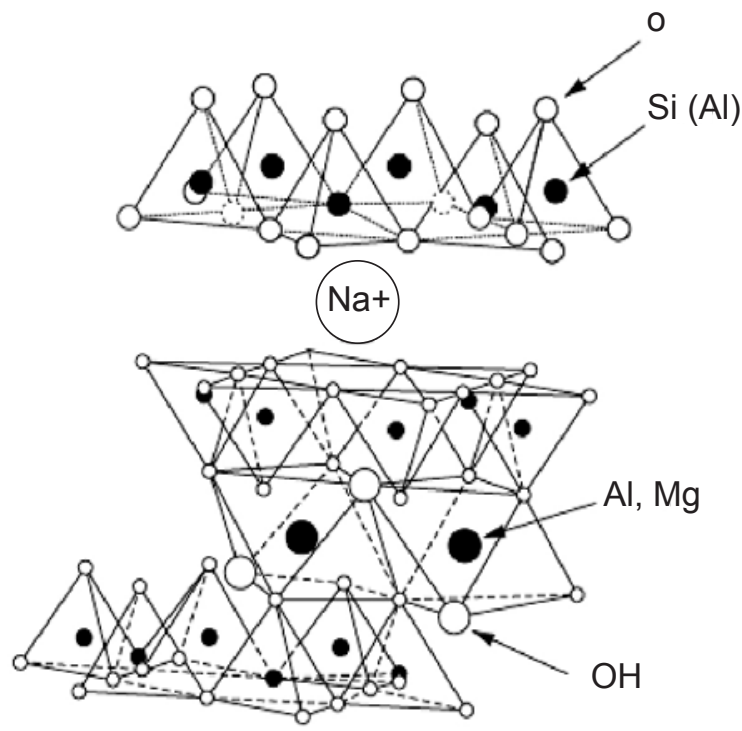

Figura 1: Estrutura química da argila bentonítica [4]. [Figure 1: Chemical structure of the bentonite clay [4].]

e tixotrópicas que apresentam, contribuindo para um melhor desempenho das propriedades dos fluidos durante a operação de perfuração de poços [5]. Os fluidos de perfuração desempenham importantes funções no processo de perfuração, como: suspensão dos detritos gerados durante a operação de perfuração, controle de pressão, estabilização das formações, lubrificação e resfriamento da broca [6].

No Brasil, as argilas comumente utilizadas no preparo de fluidos de perfuração são as argilas bentoníticas provenientes dos depósitos localizados no Município de Boa Vista, PB. Segundo os últimos dados do Departamento Nacional de Produção Mineral - DNPM [7], o volume desses depósitos corresponde a 25,3\% das reservas medidas nacionais, sendo considerado o terceiro maior jazimento do país. As argilas de Boa Vista, PB, foram descobertas no início da década de 60 e tornaramse conhecidas por suas cores características (chocolate, verde-claro, verde-lodo, vermelha e creme) e por sua capacidade de transformar-se em sódica quando tratadas com $\mathrm{Na}_{2} \mathrm{CO}_{3}$, iniciando-se, portanto, o seu processo de industrialização [2]. Essas argilas ocorrem em forma de pequenos depósitos espalhados ao longo de uma distância de aproximadamente $10 \mathrm{~km}$ [1]. As empresas mineradoras e beneficiadoras locais de bentonita utilizam as cores como guia para lavra, estocagem e beneficiamento. Além disso, as propriedades tecnológicas dessas argilas demonstram certa dependência às cores existentes e a tratamentos com agentes químicos, como $\mathrm{Na}_{2} \mathrm{CO}_{3}$ [8]. Os depósitos de bentonitas da Paraíba são explorados há mais de 40 anos e, com isso, algumas variedades de argila não são mais encontradas, a exemplo das argilas de cores vermelha e verde-claro. Nos dias atuais, podem ser encontradas em grandes quantidades as variedades das cores chocolate, verde-lodo e creme.

Atualmente, as argilas utilizadas como agente viscosificante e tixotrópico em fluidos para perfuração rotativa de poços são originadas a partir da mistura de duas ou três variedades de argila, sendo a de cor chocolate utilizada em maior quantidade por apresentar melhor resposta ao tratamento com $\mathrm{Na}_{2} \mathrm{CO}_{3}$. Por esse motivo, essa argila é classificada como uma argila de boa qualidade [2], enquanto que as demais são classificadas como argilas de qualidade inferior.

Como conseqüência do processo de mineração, os produtos industrializados apresentam queda nas suas propriedades e a maioria não atende as especificações da Petrobrás [9] para uso em fluidos de perfuração à base de água. Segundo as especificações vigentes [9], a bentonita, para ser utilizada no preparo de fluidos de perfuração à base de água, deve atender a valores mínimos dos parâmetros reológicos (propriedades reológicas) e de filtração: $\mathrm{VA} \geq 15,0 \mathrm{cP}, \mathrm{VP} \geq 4,0 \mathrm{cP}, \mathrm{LE} \leq 1,5 \mathrm{x} \mathrm{VP}$ e VF $\leq 18,0 \mathrm{~mL}$.

Para tentar melhorar a qualidade dos fluidos de perfuração preparados com essas argilas bentoníticas é freqüente a prática de aditivação do fluido, sendo comumente utilizados aditivos poliméricos. A etapa de aditivação do fluido ocorre durante a sua preparação nos tanques de lama ou mesmo durante a operação de perfuração quando é detectada a necessidade de adequação das suas propriedades [10]. Dentre os inúmeros aditivos utilizados, destacam-se os aditivos orgânicos celulósicos e sintéticos, comercialmente conhecidos por carboximetilcelulose (CMC) e poliacrilamida parcialmente hidrolisada de alto peso molecular (PAM) que atuam, dependendo das suas características, como viscosificante e/ou redutor de filtrado, lubrificante, inibidor e encapsulador de argilas hidratáveis. O CMC de cadeia longa atua aumentando a viscosidade do fluido, enquanto que o CMC de cadeia curta age, principalmente, como redutor de perda por filtração $[2,11,12]$. A PAM, por sua vez, é um excelente viscosificante, inibidor e encapsulador de argilas hidratáveis, mas não forma reboco, podendo facilitar a invasão de sólidos na formação permeável se o fluido perder a homogeneidade e flocular [13]. Dificilmente, a incorporação de um único tipo de aditivo atende às necessidades corretivas do fluido, o que torna necessário o uso de mais de um tipo de aditivo. Essa prática de aditivação demanda tempo, pois os aditivos são incorporados ao fluido um a um, e poderia ser otimizada pela adição de um composto polimérico constituído por mistura de dois ou mais polímeros. A principal razão para utilização de compostos poliméricos (composições binárias e ternárias de polímeros) é a possibilidade de combinar as propriedades de duas ou mais matériasprimas com características diferentes, visando obter um produto com propriedades adequadas, ou seja, usufruir os benefícios da mistura de polímeros, obtendo um composto que possibilite, por exemplo, o aumento das viscosidades aparente e plástica e a diminuição do 
volume de filtrado [14]

Outro ponto de grande importância é a possibilidade de melhorar, por meio da aditivação polimérica, as propriedades das argilas bentoníticas da Paraíba consideradas de qualidade inferior para utilização como agente viscosificante e tixotrópico em fluidos de perfuração e que, por este motivo, ainda são encontradas em grande quantidade [10].

Desta forma, este trabalho tem como objetivo avaliar o efeito da utilização de compostos poliméricos na aditivação de bentonitas de Boa Vista, PB, consideradas de qualidade inferior, para utilização em fluidos de perfuração à base de água, visando à melhoria das suas propriedades reológicas e de filtração.

\section{MATERIAIS}

\section{Argilas bentoníticas}

Foi estudada uma amostra de argila bentonítica sódica, composta pela mistura das argilas de cor creme e verde-lodo, conhecidas por Bofe e Verde-Lodo, respectivamente, provenientes das jazidas de Boa Vista, PB.

\section{Composto polimérico}

Para a composição do composto polimérico foram selecionadas três amostras de aditivos poliméricos: duas amostras de carboximetilcelulose de diferentes graus de viscosidade, sendo uma amostra de alta viscosidade, denominada de CMC AV, e uma amostra de baixa viscosidade, denominada CMC BV e uma amostra de poliacrilamida parcialmente hidrolisada de alto peso molecular, escolhidos a partir de estudos preliminares [15]. As amostras de CMC foram fornecidas pela Empresa Denver-Cotia Indústria e Comércio de Produtos Químicos Ltda., Cotia, SP, e a amostra de PAM foi fornecida pela Empresa System Mud Indústria e Comércio Ltda., Itajaí, SC. A Tabela I apresenta os dados técnicos dos polímeros.

\section{METODOLOGIA}

\author{
Dimensionamento de composições do composto \\ polimérico
}

As composições foram formuladas com os polímeros PAM, CMC BV e CMC AV empregando a metodologia de modelagem de misturas do planejamento experimental [16-18]. Para definir as composições foi utilizado um planejamento em rede simplex $\{3,2\}$, aumentado com pontos no interior, totalizando dez composições. As composições estão apresentadas na Tabela II. No texto, as composições serão citadas por $\mathrm{CP} 1, \mathrm{CP} 2, \mathrm{CP} 3, \mathrm{CP} 4, \mathrm{CP} 5, \mathrm{CP} 6, \mathrm{CP} 7$, CP8, CP9 e CP10.

Tabela II - Composições do composto polimérico. [Table II - The compositions of the polymeric compound.]

\begin{tabular}{cccc}
\hline Composições & $\begin{array}{c}\text { PAM } \\
(\%)\end{array}$ & $\begin{array}{c}\text { CMC BV } \\
(\%)\end{array}$ & $\begin{array}{c}\text { CMC AV } \\
(\%)\end{array}$ \\
\hline CP1 & 100 & 0 & 0 \\
CP2 & 0 & 100 & 0 \\
CP3 & 0 & 0 & 100 \\
CP4 & 50 & 50 & 0 \\
CP5 & 50 & 0 & 50 \\
CP6 & 0 & 50 & 50 \\
CP7 & 33,33 & 33,33 & 33,33 \\
CP8 & 66,66 & 16,66 & 16,66 \\
CP9 & 16,66 & 66,66 & 16,66 \\
CP10 & 16,66 & 16,66 & 66,66 \\
\hline
\end{tabular}

\section{Preparação dos fluidos de perfuração}

A preparação dos fluidos de perfuração seguiu as seguintes etapas: i) a argila bentonítica foi misturada manualmente com o composto polimérico em pó, nas composições estudadas (Tabela II); ii) essa mistura foi adicionada à água sob agitação a velocidade de 10.000 rpm;

Tabela I - Dados técnicos dos polímeros.

[Table I - Technical data of the polymers.]

\begin{tabular}{cccccc}
\hline Amostras & Função & Aplicação & Composição & Tamanho de cadeia & Viscosidade (cP) \\
\hline CMC AV & Viscosificante & Água doce & CMC de sódio & Longa & $3200^{*}$ \\
CMC BV & $\begin{array}{c}\text { Defloculante e } \\
\text { redutor de filtrado } \\
\text { Viscosificante }\end{array}$ & $\begin{array}{c}\text { Água doce } \\
\text { Água doce sódio } \\
\text { PAM salgada }\end{array}$ & $\begin{array}{c}\text { Copolímero de } \\
\text { acrilato de sódio } \\
\text { e acrilamida }\end{array}$ & Longa & $3200^{* * *}$ \\
\hline
\end{tabular}

Viscosidade medida em viscosímetro Brookfield LVF a 30 rpm: " pino 3, solução aquosa 1\% com correção de umidade; " pino 2, solução aquosa $1 \%$ com correção de umidade; " pino 2 , solução aquosa $1 \%$ com correção de umidade. 
e iii) após a adição da bentonita aditivada com o composto polimérico, o fluido permaneceu sob agitação a $17.000 \mathrm{rpm}$ durante $20 \mathrm{~min}$. Foi utilizado um agitador de alta rotação Hamilton Beach, modelo 936. Após preparação, os fluidos permaneceram em repouso durante $24 \mathrm{~h}$. Os fluidos foram preparados com concentração de $2,5 \%$ em massa de argila, ou seja, 12,5 g de argila em $500 \mathrm{~mL}$ de água deionizada. As velocidades de agitação, o tempo de agitação e o agitador são os recomendados pela norma N-2605 [19].

A mesma metodologia foi seguida para os fluidos preparados com a argila bentonítica sem aditivação.

O composto polimérico, nas composições estudadas, foi adicionado à argila bentonítica nas concentrações de $0,4 \mathrm{~g}, 0,6 \mathrm{~g}$ e $0,8 \mathrm{~g} / 12,5 \mathrm{~g}$ de argila seca. No texto estas concentrações serão tratadas por $0,4,0,6$ e $0,8 \mathrm{~g}$.

\section{Estudo reológico dos fluidos de perfuração}

O estudo reológico dos fluidos, com e sem aditivação polimérica, foi realizado determinando as viscosidades aparente (VA) e plástica (VP) e o limite de escoamento (LE), em viscosímetro Fann 35A, e o volume de filtrado em filtro prensa Fann, de acordo com a norma N-2605 [19].

\section{RESULTADOS E DISCUSSÃO}

Na Tabela III e nas Figs. 2 a 5 estão apresentados os valores de VA, VP, LE e VF para as diferentes composições estabelecidas e apresentadas na Tabela II. Os resultados mostram que, de modo geral, a aditivação polimérica da argila bentonítica melhora as propriedades reológicas e de filtração dos fluidos de perfuração comparada ao resultado do fluido preparado com a argila bentonítica sem aditivação polimérica. A aditivação da argila bentonítica com as composições CP1 (100\% de PAM) e CP3 (100\% de CMC $\mathrm{AV})$ eleva consideravelmente as viscosidades aparente (VA) e plástica (VP), desempenhando de maneira adequada a função para a qual estes polímeros são indicados. Os fluidos de perfuração preparados com a argila bentonítica aditivada com a composição CP2 (100\% de CMC BV) apresentam pequeno incremento nos valores de VA e redução significativa nos valores de VF, comparando com os resultados da argila bentonítica sem aditivo polimérico, sendo VF a propriedade que apresenta maior influência deste tipo de aditivo. Observa-se que, para a concentração de $0,4 \mathrm{~g}$ do composto polimérico, embora os valores de VP, VF e LE dos fluidos estejam de acordo com os limites especificados [9], não atendem às especificações devido aos baixos valores de VA (inferiores a $15 \mathrm{cP}$ ) que apresentam. Estes valores resultam da baixa quantidade de argila utilizada no preparo dos fluidos, o que torna necessário uma maior concentração de composto polimérico para adequação das propriedades. Para a concentração de $0,6 \mathrm{~g}$ do composto polimérico, os melhores resultados são obtidos quando a argila é aditivada com as composições CP5, CP7, CP8 e CP10, satisfazendo a todas as propriedades especificadas (VA, VP, VF e LE) [9], e, para 0,8 g de composto polimérico, as composições
Tabela III - Propriedades reológicas e de filtração dos fluidos preparados com $2,5 \%$ em massa de argila bentonítica, aditivada com as composições estabelecidas pelo delineamento de misturas. [Table III - Rheological and water loss properties of the drilling fluids prepared with $2.5 \mathrm{wt} . \%$ of bentonite clay treated with the compositions obtained through the methodology of mixture design.]

\begin{tabular}{|c|c|c|c|c|c|}
\hline \multicolumn{2}{|c|}{$\begin{array}{l}\text { Teor de Con } \\
\text { composto } \\
\text { polimérico }(\mathrm{g})\end{array}$} & $\begin{array}{l}\mathrm{VA} \\
(\mathrm{cP})\end{array}$ & $\begin{array}{l}\mathrm{VP} \\
(\mathrm{cP})\end{array}$ & $\begin{array}{l}\mathrm{VF} \\
(\mathrm{mL})\end{array}$ & $\begin{array}{c}\mathrm{LE} \\
\left(\mathrm{N} / \mathrm{m}^{2}\right)\end{array}$ \\
\hline --- & --- & 1,9 & 1,5 & 26,3 & 0,4 \\
\hline \multirow[t]{10}{*}{0,4} & CP1 & 12,3 & 7,5 & 17,0 & 4,8 \\
\hline & $\mathrm{CP} 2$ & 5,0 & 4,0 & 17,4 & 1,0 \\
\hline & CP3 & 10,3 & 6 & 17,8 & 4,3 \\
\hline & CP4 & 11,4 & 7,5 & 16,7 & 3,8 \\
\hline & CP5 & 13,8 & 8,2 & 18,6 & 5,6 \\
\hline & CP6 & 7,9 & 5,3 & 17,8 & 2,6 \\
\hline & CP7 & 12,0 & 8,0 & 16,1 & 4,0 \\
\hline & CP8 & 12,2 & 7,3 & 18,7 & 4,9 \\
\hline & CP9 & 9,7 & 6,8 & 17,2 & 2,9 \\
\hline & CP10 & 12,5 & 7,2 & 16,6 & 5,3 \\
\hline \multirow[t]{10}{*}{0,6} & $\mathrm{CP} 1$ & 13,9 & 8,3 & 16,6 & 5,6 \\
\hline & $\mathrm{CP} 2$ & 6,8 & 5,5 & 15,9 & 1,3 \\
\hline & CP3 & 14,2 & 8,8 & 17,3 & 5,4 \\
\hline & CP4 & 13,4 & 8,8 & 17,3 & 4,6 \\
\hline & CP5 & 16,7 & 10 & 17,8 & 6,7 \\
\hline & СР6 & 10,4 & 6,8 & 16,7 & 3,6 \\
\hline & CP7 & 15,3 & 9,5 & 16,9 & 5,8 \\
\hline & CP8 & 15,0 & 9,5 & 16,2 & 5,5 \\
\hline & CP9 & 12,5 & 8,5 & 17,5 & 4,0 \\
\hline & CP10 & 16,8 & 10,0 & 16,3 & 6,8 \\
\hline \multirow[t]{10}{*}{0,8} & CP1 & 15,5 & 9,0 & 16,3 & 6,5 \\
\hline & $\mathrm{CP} 2$ & 8,8 & 6,5 & 16,8 & 2,3 \\
\hline & CP3 & 17,7 & 10 & 16,4 & 7,8 \\
\hline & CP4 & 16,0 & 10,2 & 15,7 & 5,8 \\
\hline & CP5 & 20,4 & 11,5 & 16,5 & 8,9 \\
\hline & СР6 & 13,8 & 9,0 & 16,3 & 4,8 \\
\hline & CP7 & 17,8 & 10,7 & 17,1 & 7,1 \\
\hline & CP8 & 16,9 & 10,3 & 16,4 & 6,6 \\
\hline & $C P 9$ & 15,3 & 10,0 & 17,4 & 5,3 \\
\hline & CP10 & 19,9 & 11,5 & 16,6 & 8,4 \\
\hline \multicolumn{2}{|c|}{$\begin{array}{l}\text { Especificações } \\
\text { (Petrobrás, 1998) }\end{array}$} & $\geq 15,0$ & $\geq 4,0$ & $\leq 18,0$ & $\begin{array}{c}\leq 1,5 x \\
V P\end{array}$ \\
\hline
\end{tabular}

$\mathrm{CP} 1, \mathrm{CP} 3, \mathrm{CP} 4, \mathrm{CP} 5, \mathrm{CP} 7, \mathrm{CP} 8, \mathrm{CP} 9$ e CP10 atendem as especificações [9] para uso em fluidos de perfuração de poços de petróleo. Os valores de LE apresentados resultam da pequena concentração de sólidos presente nos fluidos. O teor de sólidos, cujo valor deve ser mantido no mínimo possível, é uma propriedade que deve ser controlada com rigor porque o seu aumento implica no aumento de várias outras propriedades, tais como densidade, viscosidade e forças géis, além de aumentar a probabilidade de ocorrência de problemas como desgaste dos equipamentos de circulação, 


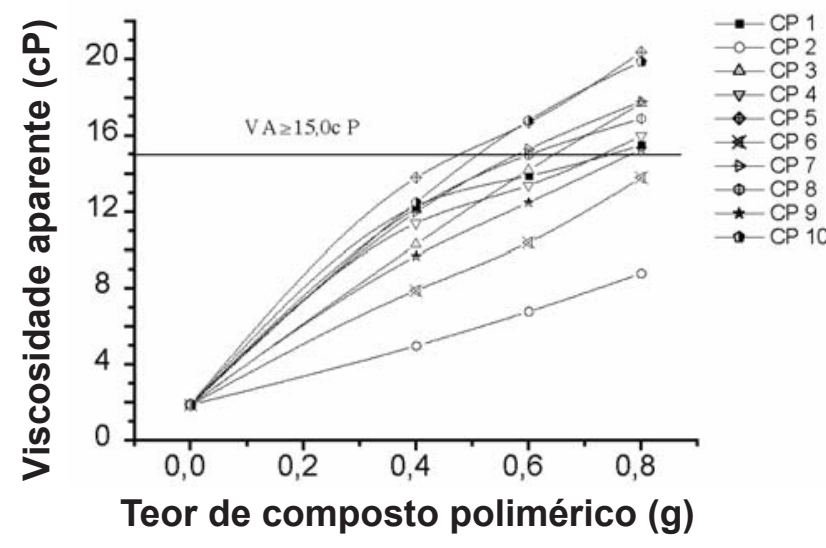

Figura 2: Viscosidade aparente dos fluidos preparados com a argila bentonítica aditivada com o composto polimérico nas composições estudadas.

[Figure 2: Apparent viscosity of the drilling fluids prepared with bentonite clay treated with the polymeric compound in the studied compositions.]

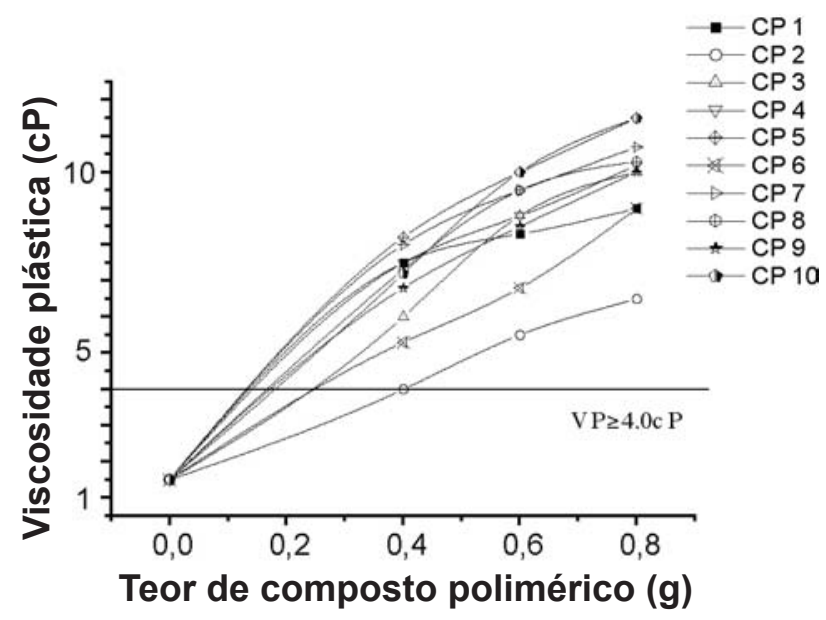

Figura 3 :Viscosidade plástica dos fluidos preparados com a argila bentonítica aditivada com o composto polimérico nas composições estudadas.

[Figure 3: Plastic viscosity of the drilling fluids prepared with bentonite clay treated with the polymeric compound in the studied compositions.]

fratura das formações devido à elevação das pressões de bombeio ou hidrostática, prisão da coluna e redução da taxa de penetração [20].

Quando os aditivos poliméricos são adicionados em meio aquoso, estes são dispersos fazendo com que a cadeia polimérica seja hidratada e assuma uma configuração que pode ser alongada ou enovelada, dependendo das características do polímero. Essa hidratação do polímero é, portanto, responsável pelo aumento da viscosidade do sistema e redução no VF. Quando se têm argilas bentoníticas, ocorre a formação de uma camada de solvatação entre as cargas negativas do polímero e as cargas positivas presentes nas arestas das partículas de argila, neutralizando-as e fazendo com que ocorra uma repulsão mútua entre elas,

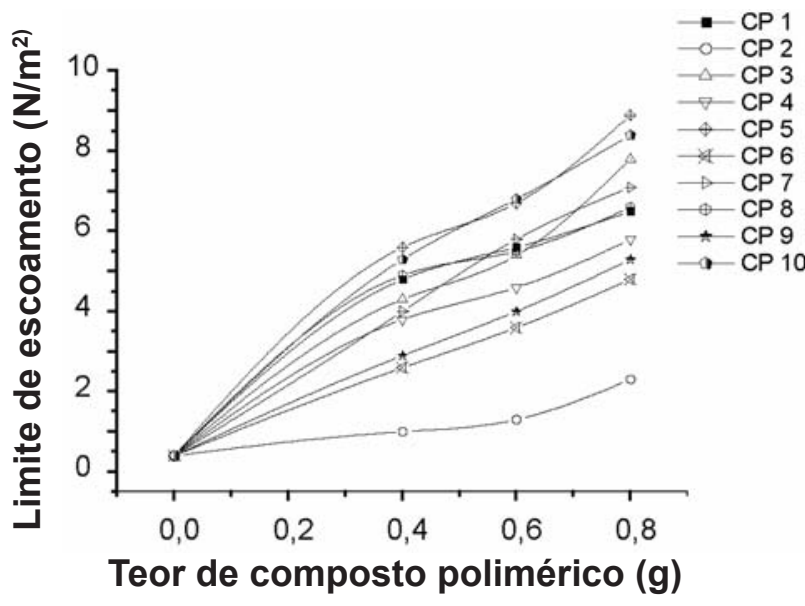

[Figura 4: Limite de escoamento dos fluidos preparados com a argila bentonítica aditivada com o composto polimérico nas composições estudadas.]

[Figure 4: Yield stress of the drilling fluids prepared with bentonite clay treated with the polymeric compound in the studied compositions.]

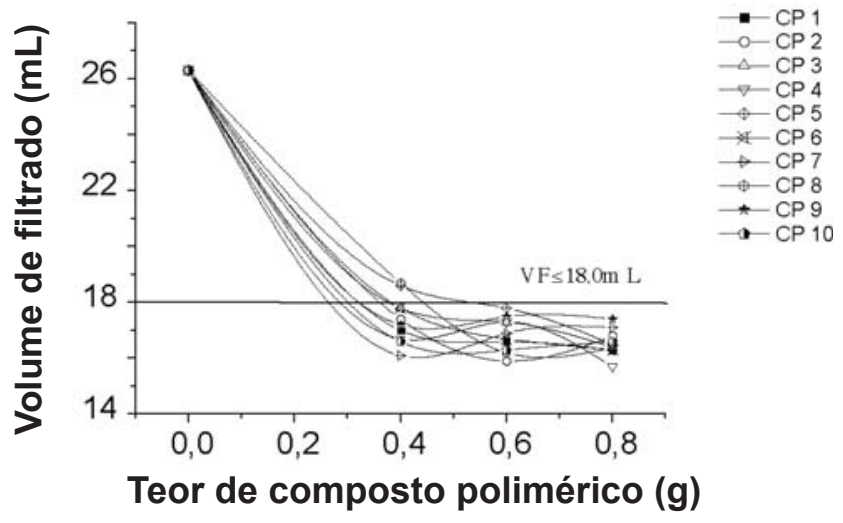

Figura 5: Volume de filtrado dos fluidos preparados com a argila bentonítica aditivada com o composto polimérico nas composições estudadas.

[Figure 5: Water loss of the drilling fluids prepared with bentonite clay treated with the polymeric compound in the studied compositions.]

tornando o sistema disperso, defloculado ou estável [2]. Esse comportamento também pode ser explicado através dos mecanismos de estabilização eletrostática e eletroestérica. A estabilização eletrostática ocorre como conseqüência da repulsão entre as cadeias do polímero ligadas às partículas de argila, enquanto que, a estabilização eletroestérica ocorre como conseqüência do aumento da distância mínima de separação entre as partículas de argila que estão envolvidas pelas cadeias poliméricas e pelas interações de caráter elétrico repulsivo entre as cadeias do polímero [21].

$\mathrm{O}$ CMC, quando em solução aquosa, libera o íon $\mathrm{Na}^{+}$, presente no grupo carboximetil $\left(-\mathrm{CH}_{2} \mathrm{COO}^{-} \mathrm{Na}^{+}\right)$tornando-se aniônico e livre para hidratar-se [13]. Desta forma, moléculas de água são adsorvidas às cadeias do polímero, que adquirem 

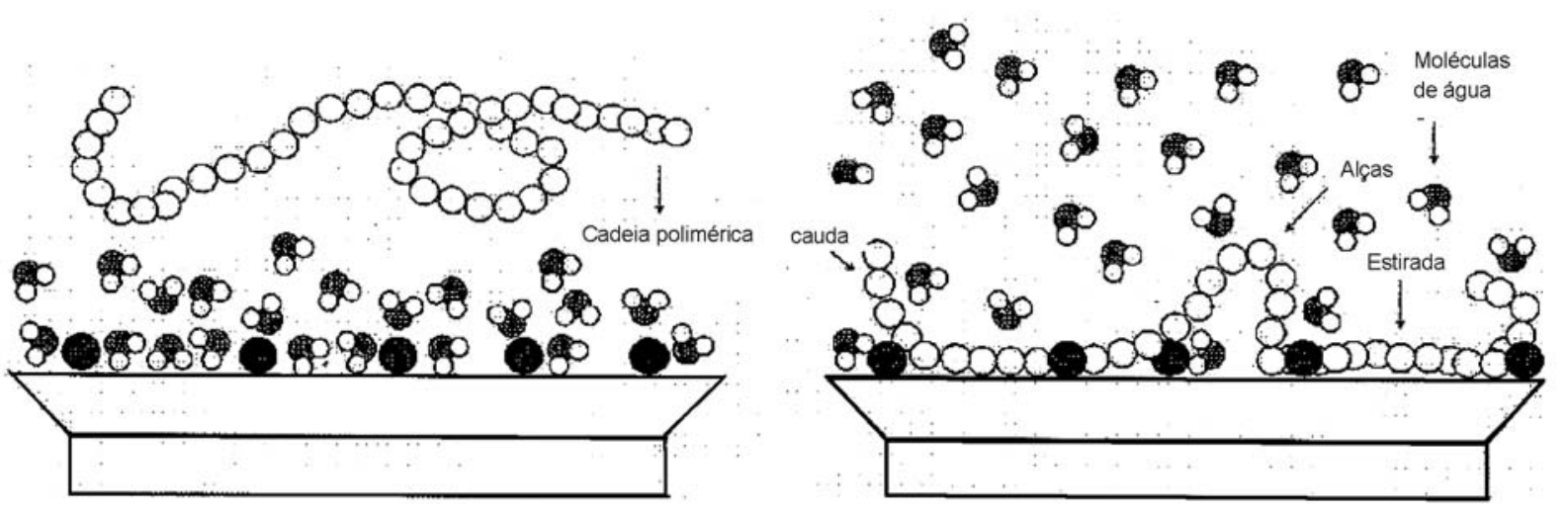

Figura 6: Representação esquemática da hidratação da cadeia polimérica e suas possíveis configurações quando adsorvidas à partícula de argila [26].

[Figure 6: Schematic representation of the polymeric chain hydration and its possible configurations when adsorbed to clay particle [26].]

uma configuração alongada e elevam a viscosidade do sistema. Esse comportamento apresenta-se diferenciado de acordo com o tamanho da cadeia do polímero. Estudos realizados mostram que polímeros de pequeno tamanho de cadeia atuam como dispersantes, enquanto que polímeros de cadeia muito longa formam estruturas tridimensionais com as partículas de argila, evidenciado pelo forte efeito exercido sobre a VA das suspensões argilosas [22].

Estudo sobre o efeito de diferentes concentrações de PAM nas propriedades reológicas de sistemas bentonitaágua, apresenta três possibilidades para as interações entre as moléculas de PAM e as partículas de argila: (i) a troca aniônica entre as hidroxilas $\left(\mathrm{OH}^{-}\right)$presentes nas superfícies das partículas de argila e os ânions carboxílicos $\left(\mathrm{COO}^{-}\right)$do polímero, (ii) a formação de ligações hidrogênio entre as hidroxilas da superfície da partícula e o grupo $\mathrm{C}=\mathrm{O}$ do polímero e (iii) o estabelecimento de pontes envolvendo íons divalentes a partir de forças eletrostáticas [23].

São três as possíveis configurações que os segmentos das cadeias de um polímero podem assumir quando em contato com partículas sólidas: estirada (trains), alça (loops) e cauda (tails) $[24,25]$. A configuração estirada caracteriza-se como uma série de segmentos consecutivos em contato com a superfície. A configuração do tipo alça consiste de segmentos em contato apenas com o meio líquido, delimitada pelas configurações do tipo estirada, enquanto que a do tipo cauda é a terminação delimitada pelo segmento com configuração do tipo alça e com movimentação livre na solução [25]. A Fig. 6 apresenta uma ilustração da hidratação da cadeia polimérica e as possíveis configurações quando adsorvidas à partícula de argila.

Comparando os resultados dos fluidos preparados com a argila bentonítica aditivada com os polímeros isolados, CP 1 (100\% de PAM), CP 2 (100\% de CMC BV) e CP 3 (100\% de CMC AV), com os resultados obtidos com os fluidos preparados com a argila bentonítica aditivada com as composições CP 4 (50\% de PAM + 50\% de CMC BV), CP 7 (33,33\% de PAM + 33,33\% de CMC BV + 33,33\% de $\mathrm{CMC}$ AV) e CP $10(16,66 \%$ de PAM + 16,66\% de CMC
$\mathrm{BV}+16,66 \%$ de CMC AV) (Tabela II, Figs. 2, 3, 4 e 5), é possível observar a influência de cada tipo de polímero sobre o comportamento reológico dos fluidos de perfuração, ou seja, que o $\mathrm{CMC} \mathrm{BV}$ atua diminuindo o $\mathrm{VF}$, enquanto o CMC AV e a PAM atuam na viscosificação dos fluidos, sendo estes comportamentos definidos de acordo com o tamanho de cadeia dos polímeros. A análise conjunta dos resultados mostra que os fluidos preparados com 2,5\% em massa de argila aditivada com $0,8 \mathrm{~g}$ das composições $\mathrm{CP}$ 1, CP 3, CP 4, CP 5, CP 7, CP 8, CP 9 e CP 10 apresentam melhor desempenho na correção/melhoria das propriedades reológicas e de filtração.

\section{CONCLUSÃO}

Os resultados deste trabalho evidenciam o benefício de se fazer uso de misturas de polímeros para obtenção de um composto com propriedades adequadas para aditivação de bentonitas, mostrando que as bentonitas classificadas como argilas de qualidade inferior, encontradas nos depósitos de Boa Vista, PB, atualmente empregadas, por exemplo, como aglomerantes para areias de fundição, podem ser utilizadas na preparação de fluidos à base de água para perfuração de poços de petróleo.

\section{AGRADECIMENTOS}

À Agência Nacional do Petróleo - ANP, ao MCT, à FINEP, ao CTBRASIL, ao CNPq/CTPetro e ao PRH-25 pelo apoio financeiro; às Empresas System Mud Indústria e Comércio Ltda. e Denver-Cotia Indústria e Comércio de Produtos Químicos Ltda. pelo fornecimento dos aditivos e ao LABDES pelo o uso de suas instalações e incentivo à pesquisa.

\section{REFERÊNCIAS}

[1] T. R. Gopinath, V. C. A. Cruz, J. A. Freire, Rev. Geologia 16, 1 (2003) 35-48. 
[2] L. V. Amorim, Melhoria, proteção e recuperação da reologia de fluidos hidroargilosos para uso na perfuração de poços de petróleo, Tese de Doutorado, UFCG, PB (2003).

[3] L. V. Amorim, E. Pereira, Bentonitas da Paraíba: O barro chega à Universidade, ABAS Informa, S. Paulo, 138 (2003).

[4] A. P. Costa Filho, A. S. Gomes, E. F. Lucas, Polímeros: Ciência e Tecnologia 15, 3 (2005) 212-217.

[5] N. J. Alderman, Proc. $3^{\text {rd }}$ Int. Symp. Chem. Oil Ind. (1988) 33-49.

[6] J. Getliff, J. Oliver, Fluido de perfuração: a força vital do poço. <www.slb.com/seed/pt/watch/mud/char.htm> acesso em dezembro de 2002.

[7] M. L. Oliveira, Bentonita, www.dnpm.gov.br, acesso em outubro de 2005.

[8] L. V. Amorim, J. D. Viana, K. V. Farias, M. I. R. Barbosa, H. C. Ferreira, Revista Matéria 11, 1 (2006) 30-40. http:// www.materia.coppe.ufrj.br/sarra/artigos/artigo10699.

[9] Petrobrás, Viscosificante para fluido de perfuração a base de água na exploração e produção de petróleo, Especificação N-2604 (1998).

[10] M. I. R. Barbosa, Desenvolvimento de aditivos poliméricos para formulação de compósitos com bentonitas, Diss. Mestrado, UFCG, PB, (2006) www.dominiopublico. gov.br/pesquisa/DetalheObraForm.do? select_action $=\& c_{-}$ obra $=34775$.

[11] L. V. Amorim, E. Pereira, C. M. Gomes, J. D. Viana, K. V. Farias, M. I. R. Barbosa, K. B. França, H. L. Lira, H. C. Ferreira, Anais do XIII Encontro Nacional de Perfuradores de Poços, Petrópolis, RJ (2003).

[12] M. I. R. Barbosa, J. D. Viana, L. V. Amorim, H. C. Ferreira, K. V. Farias, E. Pereira, Anais do XIII Congresso Brasileiro de Águas Subterrâneas, Cuiabá, MT (2004).

[13] E. Pereira, Química dos Polímeros e Aplicações - Partes I, II, III e IV, www.systemmud.com.br/index. php?pg=literatura, acesso em 8 de julho de 2002.

[14] M. I. R. Barbosa, L. V. Amorim, K. R. A. Barboza, H. C. Ferreira, Anais do XVI Congresso Brasileiro de Engenharia Química, Santos, SP (2006).

[15] M. I. R. Barbosa, Bentonitas aditivadas com polímeros para aplicação em fluidos de perfuração, Sem. Programa de Pós-Graduação em Ciência e Engenharia de Materiais, UAEMa, CCT, UFCG (2005).

[16] J. A. Cornell, Experiments with mixtures-designs, models, and the analysis of mixtures data, $2^{\text {nd }}$ Ed., Wiley (1990).

[17] L. F. A. Campos, L. V. Amorim, H. C. Ferreira, Cerâmica 52, 321 (2006) 69-75.

[18] M. I. R. Barbosa, L. V. Amorim, K. R. A. Barboza, H. C. Ferreira, Anais do Rio Oil \& Gas Expo and Conf., Rio de Janeiro (2006).

[19] Petrobrás, Argila ativada para fluido de perfuração à base de água na exploração e produção de petróleo, Método 2605 (1998).

[20] J. E. Thomas, Fundamentos de Engenharia de Petróleo, Ed. Interciência, Rio de Janeiro, Petrobrás (2001).

[21] I. R. Oliveira, A. R. Studart, R. G. Pileggi, V. C. Pandolfelli, Dispersão e empacotamento de partículas, princípios e aplicações em processamento cerâmico, Fazendo Arte Editorial, S. Paulo (2000) 30-31.

[22] H. Heller, R. Keren, Soil Sci. Am. J. 66 (2002) 19-25.

[23] N. Güngor, S. Karaoglan, Mater. Lett. 48 (2001) 168175.

[24] K. H. Sueyoshi, Propriedades coloidais e reológicas de suspensões de bentonita aditivadas com poliacrilamida e cloreto de sódio, Diss. Mestrado, UNICAMP (1994).

[25] P. F. Luckham, S. Rossi, Adv. Colloid Interface Sci. 82 (1999) 43-92.

[26] C. Breen, Appl. Clay Sci. 15 (1999) 187-219.

(Rec. 20/12/2006, Rev. 17/04/2007, Ac. 08/05/2007) 